\title{
Analysis of the antineutrino capture on protons
}

\author{
P. Vogel \\ CERN, Geneva, Switzerland \\ and California Institute of Technology, Pasadena, California 91125 \\ (Received 11 October 1983; revised manuscript received 12 December 1983)
}

\begin{abstract}
Corrections to the zeroth-approximation cross-section formula for antineutrino capture on protons at nuclear reactors are evaluated. They include recoil corrections $1 / M$ of three kinds. The true recoil correction to the cross section is negligible. The weak-magnetism-axial-vector interference is sizable, decreasing the cross section by $\sim 2 \%$ for $5-\mathrm{MeV}$ positrons. One also has to include the recoil-neutron energy in relating the positron and antineutrino energies in order to evaluate the antineutrino flux. That effect decreases the positron yield by as much as $6 \%$ for $5-\mathrm{MeV}$ positrons. The radiative corrections of order $\alpha$ have been also evaluated; they increase the cross section by $\sim 1.5 \%$ near threshold and by $\sim 0.7 \%$ at $5 \mathrm{MeV}$. Formulas and a table of numerical values of all corrections are presented.
\end{abstract}

Study of the electron-antineutrino capture on protons, $\bar{v}+p \rightarrow n+e^{+}$, is at the present time the most sensitive terrestrial test of neutrino oscillations. The accuracy, both absolute and relative, with which the positron yield is determined is fast increasing. For example, in the Caltech-SIN-TUM measurement ${ }^{1}$ at $38 \mathrm{~m}$ from the Gösgen reactor core the total statistical accuracy reached $2 \%$ and the systematic uncertainty was $5 \%$. (Besides, $1.2 \%$ uncertainty in the energy scale allows for shape distortion.)

At this level of accuracy, higher-order corrections to the zeroth approximation relating the antineutrino cross section to the neutron lifetime are becoming nonnegligible. Furthermore, they will gain in importance with the advent of future more accurate experiments. No treatment of all corrections to the cross-section formula have been made so far.

The goal of the present paper is evaluation of the accurate cross-section and positron-yield formulas, including all corrections of the order of $1 \%$, and applicable to energies encountered at nuclear reactors. In this respect, the present paper is similar, although somewhat less ambitious, to the analysis of the neutron decay by Wilkinson. ${ }^{2}$

The lowest-order cross section is obtained when one assumes that the nucleons are infinitely heavy and that the only radiative effect is the Coulomb interaction of the final proton and electron in the neutron decay. One obtains then $^{3}$

$$
\sigma_{0}\left(E_{e}\right)=\frac{2 \pi^{2} \hbar^{3}}{m_{e}{ }^{5} c^{7} f \tau_{n}} p_{e} E_{e},
$$

where $\tau_{n}$ is the neutron mean life, $f$ is the usual statistical function including the Coulomb correction for $Z=1$, and the outgoing positron and incoming antineutrino energies are related by

$$
E_{\bar{v}}=E_{e}+\left(M_{n}-M_{p}\right) c^{2} .
$$

Note that Wilkinson ${ }^{2}$ calculates $f=1.6857$ and recom- mends as an adjusted average $\tau_{n}=900 \pm 9$ sec. However, not all experimental data on $\tau_{n}$ are mutually consistent.

At the nuclear reactor one actually measures the positron yield which is in the same approximation given by

$$
Y_{0}\left(E_{e}\right)=\sigma_{0}\left(E_{e}\right) n\left(E_{\bar{v}}=E_{e}+\left(M_{n}-M_{p}\right) c^{2}\right),
$$

where $n(E)$ is the reactor antineutrino flux per unit energy.

There are several corrections to the formulas (1)-(3). The $E / M$ corrections are related either to the neglect of the neutron recoil when evaluating the cross section and antineutrino flux or to the neglect of explicit $1 / M$ terms in the weak Hamiltonian, such as weak magnetism. The other corrections, numerically of a similar magnitude, are the radiative corrections of the order $\alpha$ beyond the Coulomb effect in the statistical function $f$.

To evaluate the terms of the order $E / M$ one begins with the general matrix element of the form ${ }^{4-6}$

$$
\begin{aligned}
\mathscr{M}= & \frac{G}{\sqrt{2}}\left[\bar{u}_{n}\left[\gamma_{\mu} f-\gamma_{\mu} \gamma_{5} g-\frac{i f_{2}}{2 M} \sigma_{\mu \nu} q_{v}\right] u_{p}\right] \\
& \times\left[\bar{v}_{\bar{v}} \gamma^{\mu}\left(1-\gamma_{5}\right) v_{e}\right],
\end{aligned}
$$

where the form factors, taken as constants for our purpose, are equal to

$$
f=1, g=1.260 \pm 0.008, f_{2}=\mu_{p}-\mu_{n}=3.7 \text {. }
$$

The momentum transfer is

$$
q=p_{p}-p_{n}=p_{e}-p_{\bar{v}}
$$

and the $\gamma$ matrices and bispinors have their usual meaning. ${ }^{7}$ Note that the induced pseudoscalar does not contribute at our intended level of accuracy and that we neglect the possible second-class-current terms.

Before evaluating the cross-section formula we note that, when the recoil is included, the $\bar{v}$ and positron ener- 
gies are no longer related by Eq. (2). To obtain the positron yield one has to integrate over the recoil spectrum or, equivalently, over the positron angular distribution. The relation between the positron and antineutrino energies is (we use now $\hbar=c=1$ )

$$
E_{\bar{v}}=\frac{M\left(E_{e}+\Delta\right)+y^{2}}{M-E_{e}+p_{e} \cos \theta},
$$

where

$$
\Delta=M_{n}-M_{p}, y^{2}=\frac{\Delta^{2}-m_{e}{ }^{2}}{2} .
$$

The kinematically allowed energy interval is quite narrow, so the only modification required is the evaluation of the antineutrino flux at a somewhat larger energy than Eq. (2), taking into account the recoil kinetic energy. Remembering that the positron angular distribution is essentially isotropic $(\langle\cos \theta\rangle \sim-0.04)$, it is sufficiently accurate to use the point $\cos \theta=0$, that is,

$$
\bar{E}_{\bar{v}} \simeq E_{e}+\Delta+\frac{1}{M}\left[E_{e}\left(E_{e}+\Delta\right)+y^{2}\right] .
$$

Because the antineutrino flux $n(E)$ decreases quite rapidly with energy, Eq. (3) systematically overestimates the flux and therefore also the positron yield. Correction for this effect is represented by the quantity

$$
\delta_{\text {spec }}\left(E_{e}\right) \equiv \frac{n\left(\bar{E}_{\bar{v}}\right)-n\left(E_{e}+\Delta\right)}{n\left(\bar{E}_{\bar{v}}\right)}<0
$$

which is listed in Table I for the case of ${ }^{235} \mathrm{U}$ fission.

We now square the matrix element (4), perform the spin summations, and retain the lowest-order terms in $E / M$. The resulting cross section is written as (see also Ref. 6)

$$
\sigma\left(E_{e}\right)=\sigma_{0}\left(E_{e}\right)\left[1+\delta_{\mathrm{rec}}\left(E_{e}\right)+\delta_{\mathrm{WM}}\left(E_{e}\right)\right],
$$

where

$$
\begin{aligned}
\delta_{\mathrm{rec}}\left(E_{e}\right)=\frac{1}{f^{2}+3 g^{2}} & \left(g^{2}-f^{2}\right) \frac{\Delta}{M} \\
& \left.+(g-f)^{2} \frac{E_{e}\left(E_{e}+\Delta\right)+p_{e}{ }^{2}}{M E_{e}}\right]
\end{aligned}
$$

is the "proper" recoil correction and

$$
\delta_{\mathrm{WM}}\left(E_{e}\right)=\frac{-2 f_{2} g}{f^{2}+3 g^{2}} \frac{E_{e}+\Delta+\beta p_{e}}{M}
$$

is the correction for the weak-magnetism-axial-vector interference. (Note that there is no first-order term of the $f_{2} f$ type.) Above $p_{e}$ is the positron momentum and $\beta=p_{e} / E_{e}$. The numerical values are again listed in the table. The corrections $\delta_{\text {spec }}$ and $\delta_{\text {rec }}$, which are relatively large and strongly energy dependent, have been already included in the most recent analysis of the Gösgen reactor experiment. $^{8}$

Next we consider the radiative QED corrections of the order $\alpha$. For neutron $\beta$ decay the problem of radiative corrections has been discussed in detail by Källén, ${ }^{9}$ and in its more modern form by Sirlin. ${ }^{10}$ For our purpose we need not worry about the complications caused by the strong interactions and quark structure of the nucleon. (See, however, Ref. 10 for a discussion of these problems.) As in Refs. 2 and 11, we divide the radiative corrections into the "inner" and "outer" ones. The inner correction terms depend in our approach on the ultraviolet cutoff parameter $\Lambda$ and contain the vector-axial-vector interference term. They are, however, energy-independent constants exactly the same for the neutron decay and the antineutrino capture. Thus, we do not have to know their value as long as we express the cross section in terms of the experimental neutron lifetime. On the other hand, the outer radiative corrections will contribute an energydependent finite term to the cross-section formula and they add $\delta f=0.0254$ to the $f$ function of the neutron de$\mathrm{cay}^{2}$ in Eq. (1).

In a recent paper, ${ }^{12}$ Dicus et al. discuss the role of radiative corrections to processes changing neutrons into protons and vice versa in the context of primordial nucleosynthesis. Our results, while generally in agreement with theirs, differ in some details, mainly in the treatment of the integration over the bremsstrahlung spectrum.

The Feynman graphs contributing in order $\alpha$ to the antineutrino capture are shown in Fig. 1. In evaluating them we neglect the recoil and weak-magnetism parts of the matrix element (4). After some rather tedious algebra (see, e.g., Ref. 9), the graphs 1(a)-1(c) together contribute to the squared matrix element the quantity

$$
\begin{aligned}
\delta \mathscr{M}^{2}=\frac{\alpha}{\pi} \mathscr{M}^{2}\{ & 2 \ln \frac{\lambda}{m_{e}}\left(\frac{1}{\beta} \tanh ^{-1} \beta-1\right]-\frac{1}{\beta}\left(\tanh ^{-1} \beta\right)^{2}+\frac{3}{2} \ln \frac{M}{m_{e}}-\frac{11}{8}+\beta \tanh ^{-1} \beta+\frac{1}{\beta} L\left(\frac{2 \beta}{1+\beta}\right) \\
& \left.+\frac{1}{2\left(f^{2}+3 g^{2}\right)}\left[\ln \frac{\Lambda}{M}\left(3 f^{2}+12 f g+9 g^{2}\right)+3 g(2 f+g)\right]\right\} .
\end{aligned}
$$

Here $\mathscr{M}^{2}$ is the squared and spin-summed matrix element (4), $\lambda$ is the "photon mass" related to the infrared divergence, and $L(x)$ is the Spence function." 90 The term in square brackets represents the inner radiative corrections; the $\Lambda$-independent constant part has been chosen as in Ref. 11. These inner radiative corrections, as pointed out above, are automatically included if experimental neutron lifetime is used.
The bremsstrahlung graphs 1(d) and 1(e) give identical squared matrix elements in the neutron decay and in the antineutrino capture. In both processes they contribute only to the outer radiative corrections. In the former case one proceeds further as follows: First the integration over the photon spectrum is performed, keeping the electron energy fixed. The antineutrino energy is varied together with $E_{\gamma}$ according to the energy-conservation condition 
TABLE I. Corrections to the cross section and positron yield, Eqs. (20) and (21), in units of $10^{-3} . T_{e}$ is the positron kinetic energy in $\mathrm{MeV}$.

\begin{tabular}{rrrcr}
\hline \hline$T_{e}$ & $\delta_{\text {spec }}$ & $\delta_{\text {rec }}$ & $\delta_{\mathrm{WM}}$ & $\delta_{\text {rad }}$ \\
\hline 0.5 & -2.2 & 0.17 & -5.3 & 13.9 \\
1.0 & -3.8 & 0.18 & -7.2 & 11.7 \\
2.0 & -8.7 & 0.20 & -10.8 & 10.0 \\
3.0 & -16.8 & 0.23 & -14.4 & 8.8 \\
4.0 & -31.5 & 0.25 & -17.8 & 7.8 \\
5.0 & -56.6 & 0.27 & -21.4 & 7.1 \\
6.0 & -97.3 & 0.30 & -24.9 & 6.5 \\
\hline
\end{tabular}

$$
E_{\bar{v}}=M_{n}-M_{p}-E_{e}-E_{\gamma} .
$$

Finally, one combines the contribution for all graphs together (the infrared divergent piece disappears) and in- tegrates over the electron spectrum to obtain $\delta f=0.0254$ mentioned above.

In the antineutrino capture the situation is somewhat different. The energy-conservation condition is now

$$
E_{\bar{v}}=M_{n}-M_{p}+E_{e}+E_{\gamma}
$$

The usual detectors (such as the one in Ref. 1) will typically add the energy of the (usually soft) bremsstrahlung photon to the positron energy and register their sum. Thus, the proper procedure is to integrate over the bremsstrahlung photon spectrum keeping the antineutrino energy constant and varying the positron energy according to Eq. (15). The resulting bremsstrahlung contribution is of the form

$$
\begin{aligned}
\delta \mathscr{M}^{2}{ }_{\text {brem }}=\frac{\alpha}{\pi} \mathscr{M}^{2} & {\left.\left[2 \ln \frac{2 \omega_{M}}{\lambda}\left[\frac{1}{\beta} \tanh ^{-1} \beta-1\right]-\frac{1}{\beta}\left(\tanh ^{-1} \beta\right)^{2}+3-2 \ln \frac{2\left(\mathscr{E}+m_{e}\right)}{m_{e}}+\frac{3}{\beta} \tanh ^{-1} \beta+\frac{1}{\beta} L\right] \frac{2 \beta}{1+\beta}\right] } \\
& \left.+\frac{1}{\beta}\left[2 I_{-1}+\frac{I_{1}}{\mathscr{E}^{2}}-\frac{2 I_{0}}{\mathscr{E}}\right]\right] .
\end{aligned}
$$

Here

$$
\begin{aligned}
& \omega_{M}=E_{\bar{v}}-\left(M_{n}-M_{p}\right)-m_{e} \equiv \mathscr{E}-m_{e}, \\
& \beta=\sqrt{\mathscr{C}^{2}-m_{e}^{2}} / \mathscr{C},
\end{aligned}
$$

and

$$
\begin{aligned}
& I_{n}=\int_{1}^{\mathscr{E}} d E(\mathscr{C}-E)^{n} \ln \frac{E+p}{m_{e}}, n=1,0, \\
& I_{-1}=\int_{1}^{\mathscr{E}} \frac{d E}{\mathscr{C}-E} \ln \frac{E+p}{\mathscr{C}(1+\beta)} .
\end{aligned}
$$

The integrals $I_{i}$ can be rewritten in an approximate and rather accurate form (better than $5 \%$ for $\mathscr{C} \geq 1 \mathrm{MeV}$ ):

$$
I_{-1} \simeq L\left[1-\frac{m_{e}}{\mathscr{C}}\right]-\frac{m_{e}}{4 \mathscr{C}^{2}}\left(\mathscr{E}-m_{e}+m_{e} \ln \frac{\mathscr{C}}{m_{e}}\right],
$$

$$
\begin{aligned}
I_{0} \simeq \mathscr{E} \ln \frac{\mathscr{E}}{m_{e}}-\left(\mathscr{E}-m_{e}\right)\left(1-\ln 2+\frac{m_{e}}{4 \mathscr{E}}\right), \\
I_{1} \simeq \frac{1}{4}\left\{\left(2 \mathscr{E}^{2}+m_{e}{ }^{2}\right) \ln \frac{\mathscr{C}}{m_{e}}\right. \\
\left.+\left(\mathscr{E}-m_{e}\right)\left[\left(\mathscr{C}-m_{e}\right) 2 \ln 2-3 \mathscr{E}\right]\right\} .
\end{aligned}
$$

The Spence function can be evaluated using the expansion

$$
L(x)=\sum_{k=1}^{\infty} \frac{x^{k}}{k^{2}},|x| \leq 1 .
$$

Now we add the outer radiative part of Eq. (13) to the bremsstrahlung formula (16) and obtain the final total radiative-correction term

$$
\begin{aligned}
\delta_{\mathrm{rad}}(\mathscr{C})=\frac{\alpha}{\pi}[ & 2 \ln \frac{2\left(\mathscr{E}-m_{e}\right)}{m_{e}}\left[\frac{1}{\beta} \tanh ^{-1} \beta-1\right]-2 \ln \frac{2\left(\mathscr{C}+m_{e}\right)}{m_{e}}+\frac{3}{2} \ln \frac{M}{m_{e}}+\frac{13}{8}-\frac{2}{\beta}\left(\tanh ^{-1} \beta\right)^{2}+\frac{2}{\beta} L\left[\frac{2 \beta}{1+\beta}\right] \\
& \left.+\beta \tanh ^{-1} \beta+\frac{3}{\beta} \tanh ^{-1} \beta+\frac{1}{\beta}\left[2 I_{-1}+\frac{I_{1}}{\mathscr{E}^{2}}-\frac{2 I_{0}}{\mathscr{E}}\right]\right]
\end{aligned}
$$

It should be remembered that the $\mathscr{E}$ above represents the total positron + photon energy in the case when a real bremsstrahlung photon is emitted. The numerical value of the radiative correction factor is again shown in the table.

Thus, we have obtained the final cross-section formula

$$
\sigma(\mathscr{E})=\sigma_{0}(\mathscr{E})\left(1+\delta_{\mathrm{rec}}+\delta_{\mathrm{WM}}+\delta_{\mathrm{rad}}\right)
$$

and the positron-yield formula

$$
Y(\mathscr{E})=Y_{0}(\mathscr{E})\left(1+\delta_{\mathrm{spec}}+\delta_{\mathrm{rec}}+\delta_{\mathrm{WM}}+\delta_{\mathrm{rad}}\right) .
$$

The formulas (20) and (21) contain all corrections $\geq 0.2 \%$ for energies $\lesssim 10 \mathrm{MeV}$ relevant for the experiments at nuclear reactors. At higher energies, relevant to meson factories, one has to include the momentum-transfer depen- 


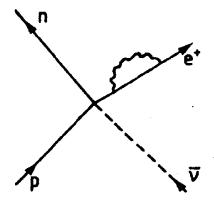

(a)

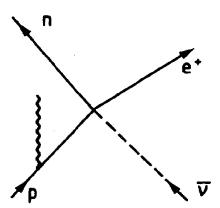

(d)

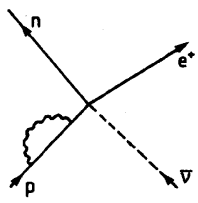

(b)

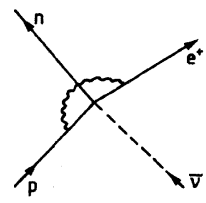

(c)
FIG. 1. Feynman graphs describing radiative corrections to the antineutrino capture on protons.

dence of the form factors $f(q)$ and $g(q)$.

Inspection of the table shows that the corrections are indeed relatively small but non-negligible for the energies of interest. The spectrum correction $\delta_{\text {spec }}$ is an exception and becomes quite sizable at higher positron and antineutrino energies. The calculated effects change in unison the shape of the positron spectrum (characterized by the ratio of yields at the boundaries) for energies between 1 and 5 $\mathrm{MeV}$ by $\sim 2.5 \%$ when only the last three terms in Eq. (21) are counted and by $\sim 8 \%$ when $\delta_{\text {spec }}$ is also included. The integrated positron yield (for positron kinetic energies $0.7-5.6 \mathrm{MeV}$ ) is decreased by $0.5 \%$ in the former cases and by $2.2 \%$ when all corrections are included.

At the present level of accuracy no significant changes in the exclusion plots of the neutrino oscillation parameters $\Delta m^{2}$ and $\sin ^{2} 2 \theta$ are expected. Indeed, calculations of the Caltech-SIN-TUM group ${ }^{13}$ confirm this conclusion. On the other hand, with increasing accuracy, these corrections will become more important.

As an illustration of the effect we show in Fig. 2 the ra-

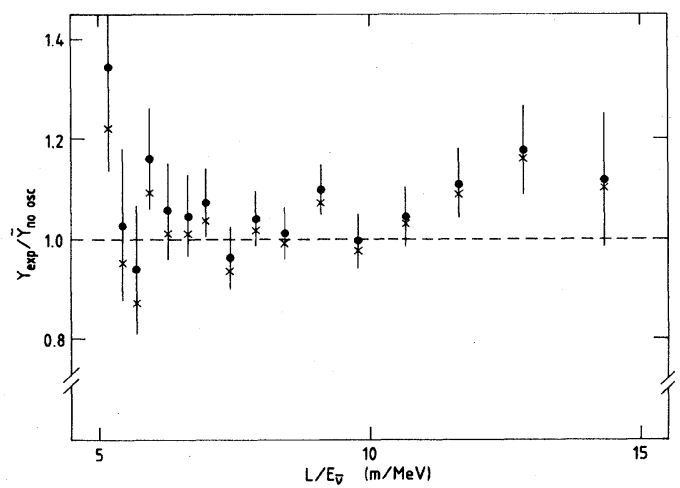

FIG. 2. Ratio of the experimental to predicted (for no neutrino oscillations) positron spectra. Experimental data and uncorrected yields $Y_{0}$ from Ref. 1; corrections are added according to Eq. (21) and the resulting ratios are denoted by full points. The original uncorrected ratios (Ref. 1, Fig. 3) are denoted by crosses. The uncertainties, shown only for the corrected ratios, are statistical at the $1 \sigma$ level. tio $Y_{\text {exp }} / Y_{\text {no osc }}$ including the corrections of Eq. (21) and using the quantities $Y_{\exp }$ and $Y_{0}$ of Table $I$ in Ref. 1. The signature of neutrino oscillations on such a graph is a deviation from a horizontal line which can be shifted away from unity due to the uncertainty in the overall normalization. These deviations in Fig. 2 are statistically insignificant for both the corrected and uncorrected points. It is of interest to note, however, that the corrected points appear to be closer to the no oscillation situation. Detailed analysis (exclusion plot), giving the largest allowed values of the oscillation parameters $\Delta m^{2}$ and $\sin ^{2} 2 \theta$, must include systematic uncertainties as well as uncertainties in the energy scale and the reader is referred to Ref. 13 for results.

\section{ACKNOWLEDGMENTS}

The author would like to thank F. Boehm, J. Gimlett, H. Kwon, B. McKellar, and J. L. Vuilleumier for valuable discussions and comments. The hospitality of CERN Theory Division is much appreciated. This work has been initiated at the Aspen Center for Physics; its stimulating atmosphere was very helpful. The work was supported in part by the U.S. Department of Energy.

\section{APPENDIX: DEVIATIONS FROM THE ALLOWED SPECTRUM SHAPE AND THE CONVERSION FROM ELECTRON TO ANTINEUTRINO SPECTRA}

The antineutrino flux $n\left(E_{\bar{v}}\right)$ for a nuclear reactor [Eq. (3)] is usually derived from the experimental electron spectrum $^{14} n_{e}\left(E_{e}\right)$ by a conversion procedure ${ }^{15,14}$ which assumes that all $\beta$ decays accompanying fission have the allowed spectrum shape $N_{0}$. That is not quite correct because in reality the spectrum is a superposition of branches of the form

$$
\begin{gathered}
N_{e}\left(E_{e}\right)=N_{0}\left(E_{e}, E_{m}\right)\left[1+c\left(E_{m}\right)+\delta_{\mathrm{QED}}\left(E_{e}, E_{m}\right)\right. \\
\left.+\left(A_{\mathrm{WM}}+A_{c}\right) E_{e}\right],
\end{gathered}
$$

where $E_{m}$ is the end-point energy and $c\left(E_{m}\right)$ is the normalization factor. [Only the main correction terms are included in Eq. (A1).] The radiative part $\delta_{\text {QED }}$ have been evaluated, e.g., in Ref. 11. The weak-magnetism slope parameter is approximately the same for all $\beta$ decays and equal to

$$
\begin{aligned}
A_{\mathrm{WM}} & =\frac{4}{3} \frac{\left\langle l+\left(\mu_{p}-\mu_{n}\right) \sigma\right\rangle}{C_{A} M\langle\sigma\rangle} \\
& \simeq \frac{4}{3} \frac{\left(\mu_{p}-\mu_{n}-\frac{1}{2}\right)}{C_{A} M} \simeq 0.5 \% / \mathrm{MeV} .
\end{aligned}
$$

The Coulomb slope factor $A_{C}$ for the Gamow-Teller transitions is

$$
A_{C} \simeq-\frac{10 Z \alpha R}{9 \hbar c} \frac{\left\langle\sigma r^{2}\right\rangle}{\langle\sigma\rangle R^{2}} \simeq-0.9 \% / \mathrm{MeV} .
$$

The last estimate in (A3) is for $Z=46$ and

$$
\left\langle\sigma r^{2}\right\rangle /\langle\sigma\rangle \simeq 0.8 R^{2} \text {, }
$$


that is, an average of the uniform and surface distribution of the decaying neutrons. The values (A2) and (A3) are, naturally, rather crude estimates.

When the experimental electron spectrum associated with fission is converted into the antineutrino spectrum $N_{\text {conv }}\left(E_{\bar{v}}\right)$ the result will deviate from the true spectrum and

$n\left(E_{\bar{v}}\right) \simeq N_{\text {conv }}\left(E_{\bar{v}}\right)\left[1+\epsilon_{\mathrm{QED}}\left(E_{\bar{v}}\right)+\epsilon_{\mathrm{WM}}\left(E_{\bar{v}}\right)+\epsilon_{C}\left(E_{\bar{v}}\right)\right]$.

(A4)
Numerically, using (A2) and (A3) together with the exact QED terms, one obtains an approximate formula valid for $2 \leq E_{\bar{v}} \leq 8 \mathrm{MeV}$,

$$
\epsilon_{\mathrm{QED}}+\epsilon_{\mathrm{WM}}+\epsilon_{C} \simeq 1.15 \times 10^{-2}\left(E_{\bar{v}}-3.0\right),
$$

where $E_{\bar{v}}$ is in $\mathrm{MeV}$. The formula (A5) makes it possible to estimate the size of errors associated with approximating the true spectrum shape by the allowed one and to make a correction for them.
1J. L. Vuilleumier et al., Phys. Lett. 114B, 298 (1982).

2D. H. Wilkinson, Nucl. Phys. A377, 474 (1982).

${ }^{3}$ E. J. Konopinski, The Theory of Beta Radioactivity (Clarendon, Oxford, 1966).

${ }^{4}$ N. Cabibbo and R. Gatto, Nuovo Cimento 15, 304 (1960).

${ }^{5}$ Y. Yamaguchi, Prog. Theor. Phys. 23, 1117 (1960).

${ }^{6}$ Ya. I. Azimov and V. M. Schekhter, Zh. Eksp. Teor. Fiz. 41, 592 (1961) [Sov. Phys. JETP 14, 424 (1962)].

7J. D. Bjorken and S. D. Drell, Relativistic Quantum Mechanics (McGraw-Hill, New York, 1965).

$8 \mathrm{~J}$. L. Gimlett, in Weak Interactions and Neutrinos, proceedings of the 8th International Workshop, Javea, Spain, 1982, edited by A. Morales (World Scientific, Singapore, 1983), p. 30; J. L. Vuilleumier, in Neutrino Mass and Gauge Structure of Weak
Interactions, proceedings of the Miniconference, Telemark, Wisconsin, 1982, edited by Vernon Barger and David Cline (AIP, New York, 1982), p. 252.

${ }^{9}$ G. Källén, Springer Tracts Mod. Phys. 46, 67 (1968).

${ }^{10}$ A. Sirlin, Revs. Mod. Phys. 50, 573 (1978).

${ }^{11}$ D. H. Wilkinson and B. E. F. Macefield, Nucl. Phys. A158, 110 (1970).

${ }^{12}$ D. A. Dicus et al., Phys. Rev. D $\underline{26}, 2694$ (1982).

${ }^{13}$ K. Gabathuler et al., Phys. Lett. (to be published).

${ }^{14} \mathrm{~K}$. Schreckenbach et al., Phys. Lett. 99B, 251 (1981); F. von Feilitzsch, A. A. Hahn, and K. Schreckenbach, ibid. 118B, 162 (1982).

${ }^{15}$ B. R. Davis, P. Vogel, F. M. Mann, and R. E. Schenter, Phys. Rev. C 19, 2259 (1979). 